\title{
Germline variants in IL4, MGMT and AKT1 are associated with prostate cancer-specific mortality: An analysis of 12,082 prostate cancer cases
}

\author{
L. M. FitzGerald ${ }^{1,2} \cdot$ S. Zhao ${ }^{3}$ - A. Leonardson ${ }^{4} \cdot$ M. S. Geybels ${ }^{4,5} \cdot$ S. Kolb ${ }^{4}$ - D. W. Lin $^{4,6}$ - J. L. Wright ${ }^{4,6}$ • \\ R. Eeles ${ }^{7,8} \cdot$ Z. Kote-Jarai ${ }^{7} \cdot$ K. Govindasami ${ }^{7} \cdot$ G. G. Giles ${ }^{2,9} \cdot$ M. C. Southey ${ }^{10} \cdot$ J. Schleutker $^{11,12} \cdot$ T. L. Tammela $^{13,14} \cdot$ \\ C. Sipeky $\mathbb{D}^{11} \cdot$ K. L. Penney ${ }^{15,16} \cdot$ M. J. Stampfer ${ }^{15,16,17} \cdot$ H. Gronberg ${ }^{18} \cdot$ F. Wiklund ${ }^{18} \cdot$ P. Stattin ${ }^{19,20} \cdot$ J. Hugosson $^{21}$. \\ D. M. Karyadi ${ }^{22}$. E. A. Ostrander 22 . Z. Feng ${ }^{23}$ - J. L. Stanford ${ }^{4,24}$
}

Received: 9 September 2017 / Revised: 9 November 2017 / Accepted: 20 November 2017 / Published online: 3 January 2018

(c) The Author(s) 2018. This article is published with open access

\begin{abstract}
Background Prostate cancer (PCa) is a leading cause of mortality and genetic factors can influence tumour aggressiveness. Several germline variants have been associated with PCa-specific mortality (PCSM), but further replication evidence is needed.

Methods Twenty-two previously identified PCSM-associated genetic variants were genotyped in seven PCa cohorts (12,082 patients; 1544 PCa deaths). For each cohort, Cox proportional hazards models were used to calculate hazard ratios and $95 \%$ confidence intervals for risk of PCSM associated with each variant. Data were then combined using a meta-analysis approach.

Results Fifteen SNPs were associated with PCSM in at least one of the seven cohorts. In the meta-analysis, after adjustment for clinicopathological factors, variants in the MGMT (rs2308327; HR 0.90; $p$-value $=3.5 \times 10^{-2}$ ) and IL4 (rs2070874; HR $1.22 ; p$-value $=1.1 \times 10^{-3}$ ) genes were confirmed to be associated with risk of PCSM. In analyses limited to men diagnosed with local or regional stage disease, a variant in $A K T 1$, rs2494750, was also confirmed to be associated with PCSM risk (HR $0.81 ; p$-value $=3.6 \times 10^{-2}$ ).

Conclusions This meta-analysis confirms the association of three genetic variants with risk of PCSM, providing further evidence that genetic background plays a role in PCa-specific survival. While these variants alone are not sufficient as prognostic biomarkers, these results may provide insights into the biological pathways modulating tumour aggressiveness.
\end{abstract}

\section{Introduction}

For men in many developed countries, prostate cancer (PCa) is the second leading cause of cancer-related deaths. While PCa mortality rates have been declining, the number of PCa deaths in Western countries is projected to be sustained for decades based on aging of the populations [1].

Traditionally, clinicians have assessed a man's likelihood of having a biologically aggressive prostate tumour using

Supplementary Information The online version of this article (https://doi.org/10.1038/s41391-017-0029-2) contains supplementary material, which is available to authorised users.

J. L. Stanford jstanfor@fredhutch.org

Extended author information available on the last page of the article clinical and pathological features assessed at diagnosis, including Gleason score, tumour stage, and serum prostatespecific antigen (PSA) level [2]. Researchers have postulated that genomic biomarkers may be able to distinguish indolent from aggressive PCa tumours, and several studies have identified tissue-based biomarkers [3, 4]. Testing primary tumour tissue for prognostic biomarkers may help identify cases at higher risk for $\mathrm{PCa}$-specific mortality (PCSM) [5] and who would benefit most from being treated aggressively early in the disease course. We postulate that it is also important to consider the host's genetic background and its potential influence on PCa outcomes.

To address this issue, our group previously assessed germline genetic variants in genes from specific biological pathways hypothesised to affect metastatic progression, to determine if genotype was related to PCSM [6]. Twenty-two PCSM-associated variants were identified in a 
Seattle-based discovery cohort, and validation in a Swedish cohort confirmed that the variants in five genes, $L E P R$, $C R Y 1, R N A S E L, I L 4$ and ARVCF, were significantly associated with PCSM. Subsequent studies provided additional evidence for replication of the $A R V C F$ variant in the Physicians' Health Study (PHS) participants with PCa [7] and variants in RNASEL, XRCC1 and AKT1 in PCa cases participating in the family-based Prostate Cancer Genetic Research Study (PROGRESS) [8]. As these prior studies had some shortcomings, including a limited number of deaths due to $\mathrm{PCa}$, we sought to further evaluate this panel of 22 SNPs in relation to PCSM in additional independent patient cohorts, and in a meta-analysis yielding greater statistical power from combining these new datasets with previously studied cohorts.

\section{Patients and methods}

\section{Study populations-new PCa cohorts}

\section{Melbourne PCa cohorts}

The Melbourne cohorts were from the Prostate Cancer Research Programme of the Cancer Council Victoria. The Melbourne Collaborative Cohort Study (MCCS) is a prospective cohort study of 41,514 participants, which has been described elsewhere [9]. The MCCS is matched to cancer registries in all Australian states and national death indices to ascertain cancer diagnoses and deaths. For this study, DNA samples were available for 1100 PCa cases, including 147 who died of PCa. The Early-Onset Prostate Cancer Family Study (EOPCFS) is a population-based family series of 1428 men diagnosed with PCa and has been described elsewhere [10]. Cases were ascertained using the population-based Victorian Cancer Registry (VCR) and 1531 unrelated cases with a DNA sample were available for this study, including 91 confirmed PCa deaths. Clinical data were obtained from the VCR and were limited to diagnosis age and Gleason score.

\section{Finnish PCa cohort}

The Finnish cohort consists of PCa cases from hereditary PCa families and from a case-control study population, described elsewhere in detail [11, 12]. All 2629 cases were of Finnish heritage. PCa diagnoses were confirmed using medical records and survival data were obtained through annual updates from the Finnish Cancer Registry. For PCa deaths $(n=281)$ identified via annual linkage to the Cancer Registry, underlying cause was confirmed using medical records.

\section{United Kingdom (UK) PCa cohort}

The UK cohort comprises men diagnosed with PCa and recruited for the UK Genetic Prostate Cancer Study (UKGPCS), which has been described elsewhere [13]. Of the 1560 cases available for this study, diagnoses and clinical-pathological data were confirmed using medical records. Vital status and cause of death were obtained from the National Health Service Information Centre and Central Register, with a total of 221 PCa-specific deaths.

\section{Study populations-previously analysed PCa cohorts}

\section{Seattle family-based PCa cohort}

The PROGRESS [14] includes cases from high-risk hereditary PCa families. Ascertainment, eligibility criteria and data collection for this study have been described previously [14, 15]. Medical records were obtained for 961 PCa cases and were used to extract clinical data on Gleason score, stage of disease, and serum PSA level at diagnosis. Death certificates confirmed underlying cause (PCSM or other), date and age at death. For this cohort, 957 cases of European ancestry had DNA available for genotyping, including 98 men who died of PCa [8].

\section{Swedish PCa cohort}

The Swedish population-based PCa cohort comprises cases enroled in Cancer of the Prostate in Sweden, which has been described elsewhere [6, 16]. For the current study, 2875 cases of European descent had DNA available for genotyping and 501 had PCa confirmed as the underlying cause of death [17]. Clinical data were obtained from the Swedish cancer registry.

\section{PHS PCa cohort}

The PHS began as a randomised, double-blind placebocontrolled trial of aspirin, and $\beta$-carotene for the prevention of cardiovascular disease and cancer and has been described in detail elsewhere [18]. The 1430 PCa cases in this study were previously chosen for a nested case-control study [19] and are restricted to self-reported Caucasians. For these analyses, 194 PCa deaths and 11 men with bone metastases were included [7]. Clinical data were abstracted from medical records.

All studies were approved by their local Institutional Review Board or Human Research Ethics Committee. Written informed consent was obtained from all study participants. 


\section{Genotyping}

Twenty-two candidate SNPs [6] were genotyped for this validation study. The MassARRAY iPLEX system (Sequenom, Inc.) was used to genotype the Swedish and Finnish samples, and 20 of the 22 SNPs in the PROGRESS samples. The remaining two SNPs (PROGRESS) and all 22 SNPs were genotyped in the Australian cohorts using TaqMan assays (Applied Biosystems). The PHS samples were genotyped using BioTrove OpenArray Technology (Applied Biosystems) and the UK samples were genotyped on the Infinium OncoArray 500K BeadChip (Illumina, Inc.). Two SNPs failed genotyping in the Swedish cohort, rs228697 and rs1029153. In the UK cohort, nine of the 22 SNPs were replaced with a surrogate SNP that was in strong linkage disequilibrium (LD; $r^{2} \geq 0.85$ ) with the original SNP (Supplementary Table 1).

Blind duplicate samples were distributed evenly across all genotyping batches from each study cohort. Concordance for the 22 SNP genotypes was $100 \%$ for the 53 Finnish duplicates, 99\% for the 24 EOPCFS duplicates, $97 \%$ for the 21 MCCS duplicates and $>93 \%$ for the 16 UK duplicates. Samples with $\geq 5$ failed SNPs were removed from further analyses $(n=49$ Finnish, $n=110$ EOPCFS, and $n=384$ MCCS cases). One Finnish case was removed due to missing follow-up data. Quality control (QC) results for the Swedish, PROGRESS and PHS studies have been reported previously [6-8]. After QC measures, 12,082 PCa cases, including 1544 confirmed PCa deaths, were available for analysis.

The minor allele frequencies (MAF) for the 22 SNPs in men who did not die of PCa from each patient cohort are shown in Supplementary Table 2. For most SNPs, the MAF is fairly similar across the cohorts with the exception of the Finnish cohort. Several SNPs in the Finnish cohort have a MAF at least $10 \%$ higher than what was found in the other cohorts, e.g., rs1137100, rs627839, rs4583514, and rs2070874. The distribution of MAF for the three SNPs associated with PCSM for each group of patients (alive, other cause of death, and PCa-specific death) for each cohort is shown in Supplementary Table 3, excluding the PHS (only summary genotyping data were available) and Swedish (missing other cause death information) cohorts.

\section{Statistical analyses}

Hazard ratios (HR), 95\% confidence intervals $(95 \% \mathrm{CI})$ and $p$-values for each SNP in relation to PCSM were calculated using Cox proportional hazards regression models for each of the seven independent cohorts. Men were followed from date of diagnosis to date of: (1) PCa-specific death; (2) death from another cause; or (3) last follow-up. Those who died of other causes and survivors were treated as censored observations. The minor allele of each SNP in the Seattlebased PCa discovery cohort was considered the "at risk" allele. For each SNP, two Cox models were tested. In the first model, both the genetic model (additive, dominant, or recessive) and clinicopathological covariates (age at diagnosis, Gleason score, stage, diagnostic PSA, and primary treatment) that were found to be significant in the original Seattle cohort were fixed [6]. In the second model, the genetic model remained fixed based on the original Seattle cohort, but the clinicopathological covariates were allowed to vary according to the best-fitting model for each cohort. Missing indicator variables were included if clinicopathological covariates had some (but not all) missing data. For both Australian cohorts, only two covariates (age at diagnosis and Gleason score) were considered in these models due to missing data.

We then performed meta-analyses to aggregate evidence across these studies using the R package, Metafor [20]. Data from the original Seattle-based discovery cohort were not included in the meta-analyses. We fitted an intercept-only linear model for each SNP, with log HRs estimated from the seven cohorts as the outcomes, and weighted by the inverse of their corresponding standard error squares. The first meta-analysis was run based on the coefficients estimated with the combination of covariates that were significant in the original Seattle cohort (first model) and the second was based on the best fitting covariates for each cohort (second model). As we were testing an a priori defined hypothesis for each SNP, an association was considered statistically significant if the nominal $p$-value was $<0.05$ (one-sided test). A one-tailed test was used because for validation we required that the effect of the risk allele on PCSM be in the same direction as in the original Seattle dataset [6].

Due to the different MAFs in the Finnish cohort and missing clinicopathological covariates in the Australian datasets, sensitivity analyses were performed where the Finnish or both the Finnish and Australian datasets were excluded. In other sensitivity analyses, men diagnosed with distant or unknown stage $\mathrm{PCa}$ were excluded due to uncertainty in defining the process of metastatic progression to lethality in such patients, and to evaluate SNP associations in men diagnosed with less advanced disease.

\section{Results}

The characteristics of the seven genotyped PCa cohorts are presented in Table 1. Overall, there were 12,082 cases with genotyping data from across the studies, of which 1544 (12.8\%) had died of PCa.

As different cohorts may have different underlying genetic susceptibilities and distributions of clinicopathological features, each cohort was first evaluated 
Table 1 Characteristics of the seven independent prostate cancer cohorts

\begin{tabular}{|c|c|c|c|c|c|c|c|}
\hline & Swedish & PROGRESS & PHS & Australia (EOPCFS) & Australia (MCCS) & Finnish & UK \\
\hline Sample size & 2875 & 957 & 1430 & 1531 & 1100 & 2629 & 1560 \\
\hline \multicolumn{8}{|l|}{ Age at diagnosis (years) } \\
\hline Mean & 65.8 & 64.5 & 70.5 & 52.9 & 67.9 & 68.6 & 61.7 \\
\hline Range & $44.6-80.4$ & $40.0-87.0$ & $45.5-100.9$ & $38.0-87.0$ & $47.0-86.0$ & $37.0-95.0$ & $36.9-88.9$ \\
\hline \multicolumn{8}{|l|}{ Follow-up time (years) } \\
\hline Mean & 6.0 & 12.73 & 11.0 & 8.1 & 8.5 & 9.1 & 3.8 \\
\hline Range & $0.3-8.6$ & $0.3-32.6$ & $0.01-27.9$ & $0.9-18.2$ & $0.01-24.5$ & $0.1-31.9$ & $0.01-11.1$ \\
\hline \multicolumn{8}{|l|}{ Age at death (years) } \\
\hline Mean & 71.2 & 80.5 & 84.2 & 61.0 & 76.4 & 77.7 & 67.7 \\
\hline Range & $48.5-85.7$ & $54.0-99.0$ & $60.9-104.3$ & $42.9-89.9$ & $53.1-91.7$ & $44.2-105.6$ & $41.7-94.2$ \\
\hline \multicolumn{8}{|l|}{ PCa-specific mortality } \\
\hline $\mathrm{No}^{\mathrm{a}}$ & $2374(82.6)$ & $782(81.7)$ & $1225(85.7)$ & $1439(94.2)$ & $953(86.6)$ & $2196(83.5)$ & $1335(85.6)$ \\
\hline Yes & $501(17.4)$ & $98(10.2)$ & $205(14.3)$ & $91(5.8)$ & $147(13.4)$ & $281(10.7)$ & $221(14.2)$ \\
\hline Unknown $^{\mathrm{b}}$ & $0(0)$ & $77(8.0)$ & $0(0)$ & $1(0.1)$ & $0(0.0)$ & $152(5.8)$ & $4(0.3)$ \\
\hline \multicolumn{8}{|l|}{ Stage } \\
\hline Local & $1885(65.6)$ & $623(65.1)$ & $1293(90.4)$ & NA & NA & $1910(72.7)$ & $967(62.0)$ \\
\hline Regional & $651(22.6)$ & $240(25.1)$ & $54(3.8)$ & NA & NA & $467(17.8)$ & $324(20.8)$ \\
\hline Distant & $266(9.3)$ & $27(2.8)$ & $54(3.8)$ & NA & NA & $203(7.7)$ & $105(6.7)$ \\
\hline Missing & $73(2.5)$ & $67(7.0)$ & $29(2.0)$ & NA & NA & $49(1.9)$ & $164(10.5)$ \\
\hline \multicolumn{8}{|l|}{ Gleason score } \\
\hline$\leq 6$ & $1375(47.8)$ & $561(58.6)$ & $662(46.3)$ & 744 (48.6) & $527(47.9)$ & $1187(45.2)$ & $627(40.2)$ \\
\hline 7 & $782(27.2)$ & $204(21.3)$ & $424(29.7)$ & $617(40.3)$ & $285(25.9)$ & 753 (28.6) & $523(33.5)$ \\
\hline $8-10$ & $467(16.2)$ & $80(8.4)$ & $192(13.4)$ & $116(7.6)$ & $146(13.3)$ & $410(15.6)$ & $289(18.5)$ \\
\hline Missing & $251(8.7)$ & $112(11.7)$ & $152(10.6)$ & $54(3.5)$ & $142(12.9)$ & $279(10.6)$ & $121(7.8)$ \\
\hline \multicolumn{8}{|c|}{ Diagnostic PSA level (ng/mL) } \\
\hline$<4$ & $148(5.1)$ & $77(8.0)$ & $116(8.1)$ & NA & NA & $217(8.3)$ & $272(17.4)$ \\
\hline $4-9.9$ & $993(34.5)$ & $360(37.6)$ & $558(39.0)$ & NA & NA & $1114(42.4)$ & $641(41.1)$ \\
\hline $10-19.9$ & $651(22.6)$ & $157(16.4)$ & $206(14.4)$ & NA & NA & $654(24.9)$ & $285(18.3)$ \\
\hline$\geq 20$ & $1003(34.9)$ & $130(13.6)$ & $136(9.5)$ & NA & NA & $574(21.8)$ & $293(18.8)$ \\
\hline Missing & $80(2.8)$ & $233(24.3)$ & $414(28.9)$ & NA & NA & $70(2.7)$ & $69(4.4)$ \\
\hline \multicolumn{8}{|l|}{ Primary therapy } \\
\hline Radical prostatectomy & $713(24.8)$ & $501(52.4)$ & $579(40.5)$ & NA & NA & $823(31.3)$ & 415 (26.6) \\
\hline Radiation therapy & $682(23.7)$ & $256(26.8)$ & $383(26.8)$ & NA & NA & $624(23.7)$ & $707(45.3)$ \\
\hline Androgen deprivation & $927(32.2)$ & $21(2.2)$ & $119(8.3)$ & NA & NA & $149(5.7)$ & $208(13.3)$ \\
\hline Active surveillance & $488(17.0)$ & $51(5.3)$ & $91(6.4)$ & NA & NA & $970(36.9)$ & 183 (11.7) \\
\hline Other & $22(0.8)$ & $42(4.4)$ & $21(1.5)$ & NA & NA & $15(0.6)$ & $47(3.0)$ \\
\hline Missing & $43(1.5)$ & $86(9.0)$ & 237 (16.6) & NA & NA & $48(1.8)$ & 0 \\
\hline
\end{tabular}

NA not available

${ }^{\text {a }}$ Died of other causes

${ }^{\mathrm{b}}$ Died but unknown cause of death

independently for associations between the 22 SNP genotypes and risk of PCSM. Fifteen SNPs were significantly associated with PCSM in at least one of the seven cohorts, and the risk alleles of four SNPs, rs1137100 (LEPR), rs2070874 (IL4), rs2494750 (AKT1), and rs5993891 $(A R V C F)$, were associated with PCSM in two of the cohorts (Supplementary Table 4).
Meta-analysis of the seven cohorts confirmed that two SNPs were associated with PCSM (Table 2). The Interleukin 4 (IL4) SNP, rs2070874, was associated with PCSM under the same genetic model adjusted for the same covariates as in the original Seattle discovery cohort (dominant; adjusted for age at diagnosis; $p=1.1 \times 10^{-2}$ ), and also when clinicopathological covariates were included in the 
Table 2 Meta-analysis results of 22 SNPs genotyped in seven prostate cancer cohorts

\begin{tabular}{|c|c|c|c|c|c|c|c|c|c|}
\hline SNP & Gene & $\begin{array}{l}\text { Risk allele } \\
\text { frequency }^{\mathrm{a}}\end{array}$ & $\begin{array}{l}\text { Discovery cohort genetic } \\
\text { model }^{\mathrm{b}} \& \text { adjustment } \\
\text { covariates }^{\mathrm{c}}\end{array}$ & $\begin{array}{l}\text { Hazard } \\
\text { ratio }^{\mathrm{d}}\end{array}$ & $95 \% \mathrm{CI}^{\mathrm{e}}$ & $p$-value $\mathrm{e}^{\mathrm{f}}$ & $\begin{array}{l}\text { Hazard } \\
\text { ratio }^{\mathrm{g}}\end{array}$ & $95 \% \mathrm{CI}^{\mathrm{e}}$ & $p$-value ${ }^{\mathrm{f}}$ \\
\hline rs1137100 & $L E P R$ & G: 0.27 & Dom-ACP & 1.01 & $0.91-1.12$ & NR & 0.98 & $0.88-1.08$ & 0.35 \\
\hline $\mathrm{rs} 228697^{\mathrm{h}}$ & PER3 & G: 0.11 & Dom-ACP & 1.33 & $1.14-1.55$ & NR & 1.24 & $1.07-1.44$ & NR \\
\hline rs635261 & RNASEL & C: 0.36 & Rec-ACP & 0.96 & $0.82-1.11$ & 0.31 & 0.92 & $0.80-1.06$ & 0.18 \\
\hline rs627839 & RNASEL & T: 0.47 & Dom-ACP & 1.05 & $0.94-1.18$ & 0.24 & 1.07 & $0.96-1.19$ & 0.17 \\
\hline rs4583514 & MSH2 & A: 0.38 & Dom-ACP & 1.03 & $0.92-1.14$ & 0.35 & 1.04 & $0.94-1.15$ & 0.28 \\
\hline rs4608577 & MSH2 & G: 0.17 & Add-A & 1.02 & $0.94-1.11$ & 0.34 & 1.03 & $0.95-1.13$ & 0.27 \\
\hline rs523349 & $S R D 5 A 2$ & G: 0.29 & Dom-A & 1.08 & $0.99-1.19$ & NR & 1.07 & $0.97-1.19$ & NR \\
\hline rs12467911 & $S R D 5 A 2$ & T: 0.28 & Dom-A & 1.10 & $1.00-1.20$ & NR & 1.09 & $0.99-1.21$ & NR \\
\hline rs11710277 & SEMA3F & G: 0.09 & Dom-ACP & 1.03 & $0.89-1.20$ & 0.35 & 0.95 & $0.83-1.09$ & NR \\
\hline rs11205 & $H S D 17 B 4$ & G: 0.39 & Rec-ACP & 1.06 & $0.92-1.21$ & NR & 1.06 & $0.93-1.21$ & NR \\
\hline rs2070874 & $I L 4$ & $\mathrm{~T}: 0.16$ & Dom-A & 1.14 & $1.04-1.26$ & 0.01 & 1.22 & $1.10-1.35$ & $1.1 \times 10^{-3}$ \\
\hline rs1799964 & $T N F$ & C: 0.21 & Dom-A & 1.07 & $0.98-1.18$ & NR & 1.06 & $0.96-1.17$ & NR \\
\hline rs4645959 & $C-M Y C$ & G: 0.04 & Add-ACP & 1.11 & $0.92-1.35$ & NR & 1.24 & $1.03-1.50$ & NR \\
\hline rs $1029153^{\mathrm{h}}$ & CXCL12 & C: 0.31 & Add-A & 1.04 & $0.95-1.14$ & NR & 1.04 & $0.95-1.14$ & NR \\
\hline rs2839685 & CXCL12 & T: 0.15 & Rec-ACP & 0.71 & $0.45-1.14$ & NR & 0.66 & $0.41-1.05$ & NR \\
\hline rs2308327 & $M G M T$ & G: 0.13 & Add-A & 0.93 & $0.84-1.02$ & 0.11 & 0.90 & $0.81-0.99$ & 0.03 \\
\hline rs10778534 & $C R Y 1$ & C: 0.36 & Dom-A & 1.03 & $0.94-1.13$ & 0.28 & 1.03 & $0.93-1.13$ & 0.32 \\
\hline rs2494750 & $A K T 1$ & G: 0.07 & Add-ACP & 0.92 & $0.79-1.08$ & 0.21 & 0.92 & $0.79-1.06$ & 0.16 \\
\hline rs1799814 & CYP1A1 & A: 0.05 & Add-ACP & 1.09 & $0.90-1.32$ & NR & 1.05 & $0.88-1.27$ & NR \\
\hline rs25487 & $X R C C 1$ & A: 0.36 & Add-A & 0.95 & $0.89-1.02$ & 0.11 & 0.94 & $0.88-1.01$ & 0.09 \\
\hline rs915927 & $X R C C 1$ & G: 0.43 & Dom-A & 1.03 & $0.94-1.14$ & 0.30 & 0.98 & $0.89-1.10$ & NR \\
\hline rs5993891 & $A R V C F$ & T: 0.05 & Dom-ACP & 0.92 & $0.76-1.12$ & 0.25 & 0.89 & $0.75-1.06$ & 0.14 \\
\hline
\end{tabular}

${ }^{\text {a }}$ Based on the Seattle prostate cancer discovery cohort

${ }^{\mathrm{b}}$ Dom dominant model; $R e c$ recessive model; Add additive model

${ }^{c}$ Adjusted for age at diagnosis (A) or age + clinicopathological (ACP) factors (Gleason score, stage, PSA, primary treatment)

${ }^{\mathrm{d}}$ Both the genetic model and adjustment covariates were fixed based on the Seattle discovery cohort

${ }^{\mathrm{e}}$ One-sided $95 \%$ confidence intervals

${ }^{\mathrm{f}} \mathrm{NR}$ not replicated (HR is in the opposite direction to the HR in the discovery cohort)

$\mathrm{g}$ The genetic model was fixed based on the Seattle discovery cohort, but the adjustment covariates vary across the seven cohorts

h These SNPs were not genotyped in the Swedish cohort

SNPs with statistically significant evidence for validation in the overall meta-analysis are shown in boldface.

model and best-fitted to each cohort $\left(p=1.1 \times 10^{-3}\right)$. The $O^{6}$-methylguanine-DNA methyltransferase (MGMT) SNP, rs2308327, was also associated with PCSM when analysed using the same genetic model as determined using the original Seattle-based cohort, but only when clinicopathological covariates were included in the model (additive; $p=3.5 \times 10^{-2}$ ). Three other SNPs, rs 228697 (PER3), rs12467911 (SRD5A2), and rs4645959 (c-MYC) were associated with PCSM in the meta-analysis, but the direction of association was opposite to that observed in the original Seattle discovery cohort, so these variants were not considered validated (Table 2).

A sensitivity analysis was performed to evaluate the SNP-PCSM associations when patients presenting with distant or unknown stage were excluded. The results for the
IL4 and MGMT SNPs were robust to this sensitivity analysis. In addition, when limiting the analysis to men diagnosed with local or regional stage there was confirmatory evidence that the SNP (rs2494750) in AKT1 was associated with PCSM under the same genetic model adjusted for the same covariates as in the original Seattle discovery cohort (additive; adjusted for clinicopathological covariates; HR = $0.81,95 \%$ CI $0.67-0.98, p=3.6 \times 10^{-2}$ ), and also when clinicopathological covariates were included in the model and best-fitted to each cohort $(\mathrm{HR}=0.83,95 \%$ CI $0.70-0.98, p=3.1 \times 10^{-2}$ ).

Other sensitivity analyses excluded the Finnish and/or Australian datasets. When the Finnish cohort was excluded the association between PCSM and rs2070874 (IL4) genotype remained significant whereas the association with 
rs2308327 (MGMT) was attenuated (Supplementary Table 5). When both Australian cohorts were excluded from the analyses, results for the ILA and MGMT SNPs were similar to those shown in Table 2. The ATK1 SNP was also associated with PCSM (HR $=0.83 ; 95 \%$ CI 0.70-0.98; $p=$ 0.04) under an additive genetic model, allowing clinicopathological covariates to vary by cohort to obtain the best-fitting model. Lastly, results for ILA and MGMT variants (Table 2) were similar after excluding the Finnish and both Australian cohorts.

\section{Discussion}

Twenty-two PCSM-associated variants were previously identified in a Seattle-based discovery cohort, yet subsequent individual replication studies have only confirmed subsets of these variants. In this large meta-analysis of $12,082 \mathrm{PCa}$ patients from seven cohorts, we confirm associations between two SNPs, rs2070874 (IL4) and rs2308327 (MGMT), and risk of PCSM. In addition, the meta-analysis highlighted an association with an $A K T 1$ SNP in the subset of men diagnosed with less advanced PCa (i.e., local or regional stage disease) or when both Australian datasets missing stage data were excluded. Findings from sensitivity analyses were robust for the ILA and MGMT SNPs, and provide supportive evidence that variants in three genes (ILA, MGMT, and AKT1) may play a role in mediating PCa aggressiveness. Previous studies have shown that $M G M T$ and $A K T 1$ variants are not associated with overall PCa risk [21-24] and while a nominal association has been observed between risk and the IL4 variant, rs2243228 [22], this variant is not linked to rs2070874 $\left(r^{2}=0.0127\right)$. However, another IL4 variant, rs2243250, which is in complete linkage disequilibrium with rs2070874, was recently associated with Gleason score 7-10 PCa in men randomised to the finasteride arm of the Prostate Cancer Prevention Trial [25]. A study in 2011 found nominal evidence to suggest that $A K T 1$ genetic variation had a possible role in relation to risk of more aggressive PCa [22], but the results were not confirmed in larger studies (i.e., OncoArray data). Collectively, these results have a number of important implications in relation to PCa outcomes. First, they support the hypothesis that underlying genetic background can influence an individual's risk of PCSM. Second, a deeper understanding of this genetic predisposition could eventually lead to early risk stratification and the discovery of therapeutic targets for treating high-risk cases. In fact, ILA, $M G M T$, and $A K T 1$ have well documented roles in carcinogenesis and they, or their receptors, have been suggested as therapeutic targets for PCa.

In the immune system, IL4, a $\mathrm{T}$ helper type $2\left(\mathrm{~T}_{\mathrm{H}} 2\right)$ cytokine, regulates the survival, growth, and differentiation of B and T lymphocytes [26], mast cells [27], and endothelial cells [28] through activation of the Type I IL4 receptor (IL4R). In tumorigenesis, studies of the effects of IL4 are conflicting; early work suggested the cytokine had anti-tumour effects [29, 30], but more recent studies have demonstrated tumorigenic effects, including the promotion of cancer cell survival and proliferation [31], greater migration and invasion [32], enhanced metabolism for tumour growth [33] and higher metastatic tumour burden [32]. In PCa, studies have shown that IL4 levels are elevated in hormone refractory disease [34], that IL4 can activate the androgen receptor when androgen is ablated or present at very low levels [35], and that overexpression of IL4 enhances the growth of androgen-sensitive LNCaP cells in androgen-deprived conditions [36]. In epithelial cancer cells, IL4 exerts its effects through the Type II IL4R (reviewed in [37]), which was found to be overexpressed in PCa cell lines, primary cultures established from fresh prostate tumours and prostate tumour specimens [38]. Notably, several therapies have been designed to target the IL4/IL4R signalling axis through its role in asthma and allergy (reviewed in [37]). While therapies specific to the Type II IL4R are still in the discovery phase, a Pseudomonas endotoxin-based IL4 chimeric protein, IL4-CTx, which targets both IL4 receptors, has been shown to cause remission of xenograft tumours developed from two PCa cell lines, DU145 and LNCaP [38]. This is particularly relevant to our finding that the rs2070874 variant of IL4 is associated with a greater risk of PCSM, and it is possible that cases carrying this variant could benefit from adjuvant treatment with emerging Type II IL4R therapies.

The MGMT protein is responsible for repair of DNA adducts generated by alkylating agents. Alkylation of DNA involves the addition of an alkyl group to the $O^{6}$-position of guanine, which induces mutation and malignant transformation due to methylguanine:thymine mispairing during DNA replication [39]. MGMT repair occurs through the covalent transfer of the alkyl group to its active site, which results in a conformational change, ubiquitination and a rapid degradation of the protein [40]. While MGMT has an important role in preventing carcinogenesis through its role in DNA repair, MGMT activity in tumours treated with chemotherapeutic $O^{6}$-alkylating agents is actually detrimental, reducing the sensitivity of the cancer cells to chemotherapy. MGMT protein levels have been shown to vary widely both within and between individuals [41], and there is evidence to suggest this is due to inherited genetic variation [42], which also alters MGMT activity [43]. Margison and colleagues [42] have shown that the variant alleles of two SNPs in perfect linkage disequilibrium, rs2308321 (I143V) and rs2308327 (K178R), are associated with a higher level of MGMT activity and are more resistant to inactivating pseudosubstrates. This may be due to more 
efficient repair of bulky adducts as a result of the rs2308321 amino acid change, which is within the MGMT-binding pocket and in close proximity to the active site C145 [43]. Here, we observed that the rs2308327 variant was associated with a reduced risk of PCSM, suggesting that inheritance of the more active protein form may protect cases from developing a high frequency of mutations in genes critical for tumorigenesis and that push the tumour toward an aggressive phenotype. However, cases carrying the rs2308327 variant may also be more resistant to chemotherapeutic $O^{6}$-alkylating agents and may benefit from concurrent treatment with an MGMT inactivator, such as lomeguatrib [44].

$A K T 1$ is a member of the AKT family of serine/threonine kinases, and within the PI3K/AKT pathway, plays a key role in cellular metabolism, growth, proliferation, differentiation, and survival $[45,46]$. The PI3K/AKT pathway also has a central function in epithelial to mesenchymal transition (EMT), a key process in tumour progression and metastasis [47]. Furthermore, alterations in the PI3K/AKT pathway have been reported in both primary and metastatic prostate tumours [48], including constitutive activation of $A K T 1$ via loss of the inhibitory phosphatase, PTEN [49-51], and the development of docetaxel resistance has been linked to this pathway in PCa patients [52]. The involvement of $A K T 1$ in cancer development and progression has made it a target for therapeutic intervention [53-55] and several Phase I and II trials, predominantly in breast cancer patients, are currently underway testing $A K T 1$ or PI3K/AKT pathway inhibitors.

Our study also illustrates how MAFs that vary substantially across populations can impact estimates of risk. This is particularly striking in the Finnish population where the MAF of several gene variants $(M S H 2, H S D 17 B 4, I L 4$, and $C X C L 12$ ) is quite different to that of the other study populations. As the ILA rs2070874 variant is more common in the Finnish PCa cohort, it may also be more frequent in the overall Finnish population, thus explaining why this variant is more strongly associated with PCSM when this cohort is removed from the meta-analysis, especially as the association may be driven by Swedish and Australian cohorts (Supplementary Tables 4 and 5). Whereas the MAF for the MGMT variant is similar across the populations and its association with PCSM is attenuated when the Finnish cohort is removed; this may be due to a loss of power as the association between MGMT and PCSM appears to be driven by all and not individual cohorts. These findings demonstrate the importance of considering underlying variant frequencies when combining data from different populations.

A limitation of our study was the level of missing clinicopathological data for some PCa patient cohorts. For example, we were unable to stratify Gleason score 7 patients into Gleason pattern $3+4$ versus $4+3$, restricting our ability to evaluate associations for these two distinct tumour grades that have different survival outcomes. We were able to exclude men with missing data on stage and men with distant stage disease, which demonstrated robust findings for $I L 4, M G M T$, and $A K T I$ variants in men diagnosed with localised or regional stage disease. It should also be noted that there was no central review of pathology slides to assign Gleason score, therefore there may have been some tumour grade misclassification across cases in these cohorts; but it is unlikely that such misclassification would differ substantially between cohorts or that it would be influenced by genotype. In addition, while all but two of the PCa cohorts had information available on primary treatment, we did not have information on secondary therapies that may have been used to treat PCa progression and could have varied between populations. There is currently no evidence that the $I L 4, M G M T$, or $A K T 1$ variant alleles alter response to therapy, and it seems unlikely that use of secondary treatment(s) by patients in these $\mathrm{PCa}$ cohorts would vary substantially by genotype. Another limitation of our study is that all of the cohorts were comprised of patients of European ancestry. Men of African ancestry have a higher $\mathrm{PCa}$ mortality rate compared to men of European ancestry [56, 57], and future studies of these genetic variants in relation to PCSM are imperative in that high-risk population.

Understanding which genetic pathways are involved in mediating PCa progression to a fatal endpoint may lead to the discovery of novel prognostic biomarkers and therapeutic targets. While the ILA, MGMT, and AKTI risk alleles confirmed in this study are insufficient as prognostic biomarkers on their own, the identification of further biomarkers in the same or similar biological pathways may lead to the development of a biomarker panel that could improve stratification of cases at diagnosis into low-risk and high-risk categories [58]. Such information could be useful to decide on initial and adjuvant treatments, clinical trial enrolment, early salvage therapy and more intensive surveillance in men at higher risk of PCSM. Furthermore, as our study suggests a common genetic susceptibility across several international $\mathrm{PCa}$ cohorts, such a panel may be relevant at a global level.

Acknowledgements The authors gratefully acknowledge that these studies were made possible by the contribution of many people, including the original investigators and the diligent teams who recruited the participants and continue to work on follow-up. We also express our gratitude to the men who took part in these studies and provided blood samples. This work was supported by awards from the Movember Foundation (to R.E. and J.L.S.) and grants from the National Cancer Institute (P50 CA097186 and K05 CA175147 to J.L. S.), with additional support from the Fred Hutchinson Cancer Research Center. The PROGRESS study was supported by grant R01 CA080122 from the National Cancer Institute and an award from the Prostate Cancer Foundation. The Finnish study was supported by 
grants from the Finnish Cancer Organisations, Sigrid Juselius Foundation, and the Academy of Finland (251074). The MCCS and EOPCFS were supported by Cancer Council Victoria and the MCCS further by the Australian National Health and Medical Research Council project grants, 940394, 126402, 209057, and 1043616. E.A. O. and D.M.K. acknowledge the Intramural Programme of the National Human Genome Research Institute. The PHS was supported by Department of Defence grants PC050569 and PC073618, National Cancer Institute grants CA-42182, CA-34944, CA-40360, and CA097193, and National Heart, Lung, and Blood Institute grants HL26490 and HL-34595. S.Z. was supported in part by the Intramural Research Programme of the NIH, National Institute of Environmental Health Sciences. K.L.P. was supported by National Research Service Awards T32 CA009001-32 and R25 CA098566. R.E. acknowledges the support of the National Institute of Health Research to the Biomedical Research Centre at The Institute of Cancer Research and Royal Marsden National Health Service Foundation Trust. The authors thank the UK Genetic Prostate Cancer Study Collaborators (Supplementary Note; www.icr.ac.uk/ukgpcs). OncoArray genotyping was funded by the US National Institutes of Health (U19 CA 148537: ELLIPSE; $\times 01 \mathrm{HG} 007492$ : Center for Inherited Disease Research, contract number HHSN268201200008I]. The PCa component of the OncoArray (PRACTICAL consortium; http://practical.ccge.medschl. cam.ac.uk/) was supported by European Commission's Seventh Framework Programme grant agreement no. 223175 (HEALTH-F2-2009223175), Cancer Research UK Grants C5047/A7357, C1287/A10118, C1287/A16563, C5047/A3354, C5047/A10692, C16913/A6135, and the National Institute of Health Cancer Post-Cancer GWAS initiative grant no. 1 U19 CA 148537-01 (the GAME-ON initiative).

Funding This work was supported by awards from the Movember Foundation (to R.E. and J.L.S.) and grants from the National Cancer Institute (P50 CA097186 and K05 CA175147 to J.L.S.), with additional support from the Fred Hutchinson Cancer Research Center.

\section{Compliance with ethical standards}

Conflict of interest The authors declare that they have no conflict of interest.

Open Access This article is licensed under a Creative Commons Attribution-NonCommercial-ShareAlike 4.0 International License, which permits any non-commercial use, sharing, adaptation, distribution and reproduction in any medium or format, as long as you give appropriate credit to the original author(s) and the source, provide a link to the Creative Commons license, and indicate if changes were made. If you remix, transform, or build upon this article or a part thereof, you must distribute your contributions under the same license as the original. The images or other third party material in this article are included in the article's Creative Commons license, unless indicated otherwise in a credit line to the material. If material is not included in the article's Creative Commons license and your intended use is not permitted by statutory regulation or exceeds the permitted use, you will need to obtain permission directly from the copyright holder. To view a copy of this license, visit http://creativecommons. org/licenses/by-nc-sa/4.0/.

\section{References}

1. Bray F, Soerjomataram I. The changing global burden of cancer: transitions in human development and implications for cancer prevention and control. In: Gelband $\mathrm{H}$, Jha P, Sankaranarayanan $\mathrm{R}$, Horton $\mathrm{S}$ (eds) Cancer: disease control priorities. The
International Bank for Reconstruction and Development/The World Bank. Washington, DC; 2015.

2. Eifler JB, Feng Z, Lin BM, Partin MT, Humphreys EB, Han M, et al. An updated prostate cancer staging nomogram (Partin tables) based on cases from 2006 to 2011. BJU Int. 2013;111:22-9.

3. Martin NE. New developments in prostate cancer biomarkers. Curr Opin Oncol. 2016;28:248-52.

4. Moschini M, Spahn M, Mattei A, Cheville J, Karnes RJ. Incorporation of tissue-based genomic biomarkers into localized prostate cancer clinics. BMC Med. 2016;14:67.

5. Ross AE, D'Amico AV, Freedland SJ. Which, when and why? Rational use of tissue-based molecular testing in localized prostate cancer. Prostate Cancer Prostatic Dis. 2016;19:1-6.

6. Lin DW, FitzGerald LM, Fu R, Kwon EM, Zheng SL, Kolb S, et al. Genetic variants in the LEPR, CRY1, RNASEL, IL4, and ARVCF genes are prognostic markers of prostate cancer-specific mortality. Cancer Epidemiol Biomark Prev. 2011;20:1928-36.

7. Penney KL, Shui IM, Feng Z, Sesso HD, Stampfer MJ, Stanford JL. Replication of a genetic variant for prostate cancer-specific mortality. Prostate Cancer Prostatic Dis. 2015;18:260-3.

8. Karyadi DM, Zhao S, He Q, McIntosh L, Wright JL, Ostrander EA, et al. Confirmation of genetic variants associated with lethal prostate cancer in a cohort of men from hereditary prostate cancer families. Int J Cancer. 2015;136:2166-71.

9. Giles G, English D. The Melbourne Collaborative Cohort Study. IARC Sci Publ. 2002;156:69-70.

10. Eeles RA, Olama AA, Benlloch S, Saunders EJ, Leongamornlert DA, Tymrakiewicz M, et al. Identification of 23 new prostate cancer susceptibility loci using the iCOGS custom genotyping array. Nat Genet. 2013;45:385-91. 391e381-2

11. Laitinen VH, Wahlfors T, Saaristo L, Rantapero T, Pelttari LM, Kilpivaara O, et al. HOXB13 G84E mutation in Finland: population-based analysis of prostate, breast, and colorectal cancer risk. Cancer Epidemiol Biomark Prev. 2013;22:452-60.

12. Schleutker J, Matikainen M, Smith J, Koivisto P, Baffoe-Bonnie A, Kainu T, et al. A genetic epidemiological study of hereditary prostate cancer (HPC) in Finland: frequent HPCX linkage in families with late-onset disease. Clin Cancer Res. 2000;6:4810-5.

13. Eeles RA. Genetic predisposition to prostate cancer. Prostate Cancer Prostatic Dis. 1999;2:9-15.

14. Stanford JL, FitzGerald LM, McDonnell SK, Carlson EE, McIntosh LM, Deutsch K, et al. Dense genome-wide SNP linkage scan in 301 hereditary prostate cancer families identifies multiple regions with suggestive evidence for linkage. Hum Mol Genet. 2009;18:1839-48.

15. Janer M, Friedrichsen DM, Stanford JL, Badzioch MD, Kolb S, Deutsch K, et al. Genomic scan of 254 hereditary prostate cancer families. Prostate. 2003;57:309-19.

16. Van Hemelrijck M, Wigertz A, Sandin F, Garmo H, Hellstrom K, Fransson P, et al. Cohort profile: the National Prostate Cancer Register of Sweden and Prostate Cancer data Base Sweden 2.0. Int J Epidemiol. 2013;42:956-67.

17. World Health Organisation. Manual of the international statistical classification of diseases, injuries, and causes of death. Geneva: World Health Organisation; 1977.

18. Steering Committee of the Physicians' Health Study Research Group. Final report on the aspirin component of the ongoing Physicians' Health Study. N Engl J Med. 1989;321:129-35.

19. Penney KL, Schumacher FR, Li H, Kraft P, Morris JS, Kurth T, et al. A large prospective study of SEP15 genetic variation, interaction with plasma selenium levels, and prostate cancer risk and survival. Cancer Prev Res. 2010;3:604-10.

20. Baujat B, Mahe C, Pignon JP, Hill C. A graphical method for exploring heterogeneity in meta-analyses: application to a metaanalysis of 65 trials. Stat Med. 2002;21:2641-52. 
21. Agalliu I, Kwon EM, Salinas CA, Koopmeiners JS, Ostrander EA, Stanford JL. Genetic variation in DNA repair genes and prostate cancer risk: results from a population-based study. Cancer Causes Control. 2010;21:289-300.

22. Kwon EM, Salinas CA, Kolb S, Fu R, Feng Z, Stanford JL, et al. Genetic polymorphisms in inflammation pathway genes and prostate cancer risk. Cancer Epidemiol Biomark Prev. 2011;20:923-33.

23. Loh YH, Mitrou PN, Bowman R, Wood A, Jeffery H, Luben RN, et al. MGMT Ile143Val polymorphism, dietary factors and the risk of breast, colorectal and prostate cancer in the European Prospective Investigation into Cancer and Nutrition (EPIC)-Norfolk study. DNA Repair. 2010;9:421-8.

24. Ritchey JD, Huang WY, Chokkalingam AP, Gao YT, Deng J, Levine $\mathrm{P}$, et al. Genetic variants of DNA repair genes and prostate cancer: a population-based study. Cancer Epidemiol Biomark Prev. 2005;14:1703-9.

25. Winchester DA, Till C, Goodman PJ, Tangen CM, Santella RM, Johnson-Pais TL, et al. Association between variants in genes involved in the immune response and prostate cancer risk in men randomized to the finasteride arm in the Prostate Cancer Prevention Trial. Prostate. 2017;77:908-19.

26. Defrance T, Vanbervliet B, Aubry JP, Takebe Y, Arai N, Miyajima A, et al. B cell growth-promoting activity of recombinant human interleukin 4. J Immunol. 1987;139:1135-41.

27. Mosmann TR, Bond MW, Coffman RL, Ohara J, Paul WE. T-cell and mast cell lines respond to B-cell stimulatory factor 1 . Proc Natl Acad Sci USA. 1986;83:5654-8.

28. Toi M, Harris AL, Bicknell R. Interleukin-4 is a potent mitogen for capillary endothelium. Biochem Biophys Res Commun. 1991;174:1287-93.

29. Lahm H, Schnyder B, Wyniger J, Borbenyi Z, Yilmaz A, Car BD, et al. Growth inhibition of human colorectal-carcinoma cells by interleukin-4 and expression of functional interleukin-4 receptors. Int J Cancer. 1994;59:440-7.

30. Volpert OV, Fong T, Koch AE, Peterson JD, Waltenbaugh C, Tepper RI, et al. Inhibition of angiogenesis by interleukin 4. J Exp Med. 1998;188:1039-46.

31. Hallett MA, Venmar KT, Fingleton B. Cytokine stimulation of epithelial cancer cells: the similar and divergent functions of IL-4 and IL-13. Cancer Res. 2012;72:6338-43.

32. DeNardo DG, Barreto JB, Andreu P, Vasquez L, Tawfik D, Kolhatkar N, et al. CD4(+) T cells regulate pulmonary metastasis of mammary carcinomas by enhancing protumor properties of macrophages. Cancer Cell. 2009;16:91-102.

33. Venmar KT, Kimmel DW, Cliffel DE, Fingleton B. IL4 receptor alpha mediates enhanced glucose and glutamine metabolism to support breast cancer growth. Biochim Biophys Acta. 2015;1853:1219-28.

34. Wise GJ, Marella VK, Talluri G, Shirazian D. Cytokine variations in patients with hormone treated prostate cancer. J Urol. 2000;164:722-5.

35. Lee SO, Lou W, Hou M, Onate SA, Gao AC. Interleukin-4 enhances prostate-specific antigen expression by activation of the androgen receptor and Akt pathway. Oncogene. 2003;22:7981-8.

36. Lee SO, Pinder E, Chun JY, Lou W, Sun M, Gao AC. Interleukin4 stimulates androgen-independent growth in LNCaP human prostate cancer cells. Prostate. 2008;68:85-91.

37. Bankaitis KV, Fingleton B. Targeting IL4/IL4R for the treatment of epithelial cancer metastasis. Clin Exp Metastasis. 2015;32:847-56.

38. Husain SR, Kawakami K, Kawakami M, Puri RK. Interleukin-4 receptor-targeted cytotoxin therapy of androgen-dependent and -independent prostate carcinoma in xenograft models. Mol Cancer Ther. 2003;2:245-54.
39. Margison GP, Santibanez Koref MF, Povey AC. Mechanisms of carcinogenicity/chemotherapy by O6-methylguanine. Mutagenesis. 2002;17:483-7.

40. Xu-Welliver M, Pegg AE. Degradation of the alkylated form of the DNA repair protein, $\mathrm{O}(6)$-alkylguanine-DNA alkyltransferase. Carcinogenesis. 2002;23:823-30.

41. Janssen K, Eichhorn-Grombacher U, Schlink K, Nitzsche S, Oesch F, Kaina B. Long-time expression of DNA repair enzymes MGMT and APE in human peripheral blood mononuclear cells. Arch Toxicol. 2001;75:306-12.

42. Margison GP, Heighway J, Pearson S, McGown G, Thorncroft MR, Watson AJ, et al. Quantitative trait locus analysis reveals two intragenic sites that influence O6-alkylguanine-DNA alkyltransferase activity in peripheral blood mononuclear cells. Carcinogenesis. 2005;26:1473-80.

43. Mijal RS, Kanugula S, Vu CC, Fang Q, Pegg AE, Peterson LA. DNA sequence context affects repair of the tobacco-specific adduct $\mathrm{O}(6)$-[4-Oxo-4-(3-pyridyl)butyl]guanine by human $\mathrm{O}(6)$ alkylguanine-DNA alkyltransferases. Cancer Res. 2006;66: 4968-74.

44. Watson AJ, Sabharwal A, Thorncroft M, McGown G, Kerr R, Bojanic S, et al. Tumor $\mathrm{O}(6)$-methylguanine-DNA methyltransferase inactivation by oral lomeguatrib. Clin Cancer Res. 2010;16:743-9.

45. Cheung M, Testa JR. Diverse mechanisms of AKT pathway activation in human malignancy. Curr Cancer Drug Targets. 2013;13:234-44.

46. Pal I, Mandal M. PI3K and Akt as molecular targets for cancer therapy: current clinical outcomes. Acta Pharmacol Sin. 2012;33:1441-58.

47. Larue L, Bellacosa A. Epithelial-mesenchymal transition in development and cancer: role of phosphatidylinositol 3' kinase/ AKT pathways. Oncogene. 2005;24:7443-54.

48. Taylor BS, Schultz N, Hieronymus H, Gopalan A, Xiao Y, Carver $\mathrm{BS}$, et al. Integrative genomic profiling of human prostate cancer. Cancer Cell. 2010;18:11-22.

49. Davies MA, Koul D, Dhesi H, Berman R, McDonnell TJ, McConkey D, et al. Regulation of Akt/PKB activity, cellular growth, and apoptosis in prostate carcinoma cells by MMAC/ PTEN. Cancer Res. 1999;59:2551-6.

50. Stambolic V, Suzuki A, de la Pompa JL, Brothers GM, Mirtsos C, Sasaki $\mathrm{T}$, et al. Negative regulation of PKB/Akt-dependent cell survival by the tumor suppressor PTEN. Cell. 1998;95: 29-39.

51. Sun H, Lesche R, Li DM, Liliental J, Zhang H, Gao J, et al. PTEN modulates cell cycle progression and cell survival by regulating phosphatidylinositol 3,4,5,-trisphosphate and Akt/protein kinase B signaling pathway. Proc Natl Acad Sci USA. 1999;96:6199-204.

52. McCubrey JA, Steelman LS, Kempf CR, Chappell WH, Abrams SL, Stivala F, et al. Therapeutic resistance resulting from mutations in Raf/MEK/ERK and PI3K/PTEN/Akt/mTOR signaling pathways. J Cell Physiol. 2011;226:2762-81.

53. Bitting RL, Armstrong AJ. Targeting the PI3K/Akt/mTOR pathway in castration-resistant prostate cancer. Endocr Relat Cancer. 2013;20:R83-99.

54. Courtney KD, Corcoran RB, Engelman JA. The PI3K pathway as drug target in human cancer. J Clin Oncol. 2010;28:1075-83.

55. Nitulescu GM, Margina D, Juzenas P, Peng Q, Olaru OT, Saloustros E, et al. Akt inhibitors in cancer treatment: The long journey from drug discovery to clinical use (Review). Int J Oncol. 2016;48:869-85.

56. DeSantis CE, Siegel RL, Sauer AG, Miller KD, Fedewa SA, Alcaraz KI, et al. Cancer statistics for African Americans, 2016: progress and opportunities in reducing racial disparities. CA Cancer J Clin. 2016;66:290-308. 
57. Mahal BA, Aizer AA, Ziehr DR, Hyatt AS, Choueiri TK, Hu JC, et al. Racial disparities in prostate cancer-specific mortality in men with low-risk prostate cancer. Clin Genitourin Cancer. 2014;12: e189-195.
58. Seymour CW, Cooke CR, Wang Z, Kerr KF, Yealy DM, Angus DC, et al. Improving risk classification of critical illness with biomarkers: a simulation study. J Crit Care. 2013;28:541-8.

\section{Affiliations}

L. M. FitzGerald (i) ${ }^{1,2} \cdot$ S. Zhao ${ }^{3} \cdot$ A. Leonardson ${ }^{4} \cdot$ M. S. Geybels ${ }^{4,5} \cdot$ S. Kolb ${ }^{4}$ - D. W. Lin ${ }^{4,6} \cdot$ J. L. Wright ${ }^{4,6}$.

R. Eeles $\mathbb{D}^{7,8} \cdot$ Z. Kote-Jarai ${ }^{7} \cdot$ K. Govindasami ${ }^{7} \cdot$ G. G. Giles ${ }^{2,9} \cdot$ M. C. Southey ${ }^{10} \cdot$ J. Schleutker ${ }^{11,12} \cdot$ T. L. Tammela ${ }^{13,14}$.

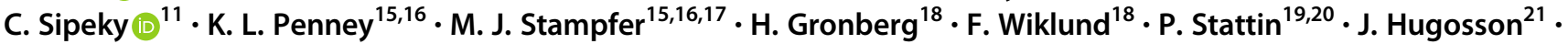
D. M. Karyadi ${ }^{22}$ - E. A. Ostrander ${ }^{22} \cdot$ Z. Feng ${ }^{23}$ J. L. Stanford ${ }^{4,24}$

1 Cancer, Genetics and Immunology, Menzies Institute for Medical Research, University of Tasmania, Hobart, TAS 7001, Australia

2 Cancer Epidemiology and Intelligence Division, Cancer Council Victoria, Melbourne, VIC 3004, Australia

3 Biostatistics \& Computational Biology Branch, National Institute of Environmental Health Science, Research Triangle Park, NC 27709, USA

4 Fred Hutchinson Cancer Research Center, Division of Public Health Science, Seattle, WA 98109, USA

5 Department of Epidemiology, GROW School for Oncology and Developmental Biology, Maastricht University Medical Centre, 6211 LK Maastricht, The Netherlands

6 Department of Urology, University of Washington, School of Medicine, Seattle, WA 98195, USA

7 The Institute of Cancer Research, Sutton SM2 5NG, UK

8 Royal Marsden National Health Service Foundation Trust, London and Sutton SW3 6JJ, UK

9 Centre for Epidemiology and Biostatistics, Melbourne School of Population and Global Health, University of Melbourne, Carlton, VIC 3053, Australia

10 Genetic Epidemiology Laboratory, Department of Pathology, University of Melbourne, Parkville, VIC 3010, Australia

11 Department of Medical Biochemistry and Genetics, Institute of Biomedicine, 20014 University of Turku, Finland

12 Tuch Microbiology and Genetics, Department of Medical Genetics, Turku University Hospital, 20520 Turku, Finland
13 Prostate Cancer Research Center, School of Medicine, University of Tampere, 33100 Tampere, Finland

14 Department of Urology, Tampere University Hospital, 33521 Tampere, Finland

15 Channing Division of Network Medicine, Department of Medicine, Brigham and Women's Hospital and Harvard Medical School, Boston, MA 02115, USA

16 Department of Epidemiology, Harvard T.H. Chan School of Public Health, Boston, MA 02115, USA

17 Department of Nutrition, Harvard T.H. Chan School of Public Health, Boston, MA 02115, USA

18 Department of Medical Epidemiology and Biostatistics, Karolinska Institute, 17177 Stockholm, Sweden

19 Department of Surgical Sciences, Uppsala University, 75236 Uppsala, Sweden

20 Department of Surgical and Perioperative Sciences, Urology and Andrology, Umeå University, 90187 Umeå, Sweden

21 Department of Urology, Institute of Clinical Sciences, University of Göteborgs, 40530 Göteborgs, Sweden

22 National Human Genome Research Institute, National Institutes of Health, Bethesda, MD 20854, USA

23 Department of Biostatistics, MD Anderson Cancer Center, Houston, TX 77030, USA

24 Department of Epidemiology, School of Public Health, University of Washington, Seattle, WA 98195, USA 